\title{
Moving from One Site to Multi-Site Vulnerability Studies: Decision Analysis for Trade-off Between Risks and Benefits
}

\author{
S. M. Hoseyni ${ }^{1 *}$ and A. Calissano ${ }^{2}$ \\ 1. Energy Department, Politecnico di Milano, Milan, Italy \\ 2. Department of Mathematics, Politecnico di Milano, Milan, Italy
}

\begin{abstract}
This paper provides a vulnerability study for a gas production site. Decision tree analysis is implemented to provide risk-informed decisions on the maintenance strategies to minimize the unavailability and downtime costs. In this context, the decision analysis assesses the impact on the availability of the plant after having decided to buy some spare parts to mitigate the criticality of some equipment. This will help the decision makers to make an optimal decision before spending their budget on buying the spare parts while they can reliably forecast a maximized availability. The decision analysis also will move from one site approach to the multi-site vulnerability studies. Result concludes a recommendation for the company on deciding the optimum maintenance strategies and provides a list of required spare parts to be bought in advance to reduce the associated risks and costs.
\end{abstract}

Keyword: Decision Analysis; Maintenance Decision; Risk; Vulnerability.

\section{Introduction}

The ultimate task for an engineer is to establish a consistent decision basis for planning, design, manufacturing, construction, operation and management of engineering facilities such that the overall life cycle benefit of the facilities is maximized [1]. In this context, international companies that sell their products and assure the availability of their systems want to minimize the downtime and maximize the availability $[2,3,4]$. They must decide on the optimal maintenance strategies to keep their system operational while the safety and reliability besides the resilience are crucially considered [5]. There must be an optimal trade-off between achieving the best maintenance strategy with the minimized cost and the system reliability with maximized safety [6]. The challenging issue in making optimized decisions is that usually the realistic results of the decision taken is observed after the decision is, actually, taken and implemented on the industrial system [7]. Therefore, a framework that reliably predict the outcome of the decision that will be taken is very promising [8].

Decision analysis, or applied decision theory, was first developed by Raffia and Schlaifer [9] to apply the theory of decision making in uncertain environments, developed by the work of great $18^{\text {th }}$ centuries scientists namely Bernoulli, Bayes, and Laplace, on real-world applications enabling the decision makers apply what she/he is asked for on a system that is provided with uncertain information [10]. Nowadays, the decision theory is applied on different fields of study like engineering [11, 12, 13], medical science [14] to business and economics [15]. Recent advancements of decision analysis in engineering field is on its application on structural health monitoring [16, 17] and sensor placement and scheduling [18, 19, 20]

In this work, we will show an application of decision analysis on the maintenance of a chemical plant that produces industrial gas. The case study presented is about the maintenance decisions in the plants and raises the question on the maintenance strategies to decide on the best action in order to decrease company's risk of operational failures. These failures may result in safety concerns, prolonged downtime (availability), or repeated trips (reliability). It is aimed at establishing recommendations (maintenance, spare parts) for each of the components. These recommendations will define when and why it is convenient to buy spare components

1. Corresponding author Email: seyedmojtaba.hoseyni@polimi.it 
in advance, considering the failure risk of the plants, the recovery time and the cost of spare and unavailability.

To answer these questions, a risk model is developed. The decision analysis method [21] is used for this purpose. Decision trees are drawn for each subcomponents of the plant, and the expected value of each maintenance strategy are calculated. The decision analysis is performed in two approaches of one site and multi-site plant. In multi-site approach the expected values are computed considering that a fraction of production of failed plant can be replaced by another plant's product. Based on these results, maintenance recommendations are provided to the company.

This paper is organized as follows:

At section 2, decision tree analysis is provided. The decision tree analysis for one site and multi-site approach and maintenance recommendations are discussed with details in this section. Section 3 provides the concluding remarks.

\section{Decision Tree Analysis}

Decision trees are special types of event trees which are used as a decision support tool to apply the framework of decision theory to provide a theoretical and figurative way for risk analysis and maintenance decisions [22]. A decision tree is a model of decisions scenarios along with all associated possible consequences, costs and utilities [23]. This tree has a structure like a flowchart in which each node represents a possible action, each branch represents the outcome of that action and each leaf represents a label (i.e. decision taken after considering all the actions). Visualizations that are provided by the decision trees greatly enhance the set of potential actions/decisions and their associated consequences in a unified traceable manner. In the decision tree analysis, the consequences of each possible action are defined in monetary terms (utility) [24]. Optimal decision is then identified as the decision with the maximum expected value of that utility function. The utility function expresses the decision-maker's preferences with reference to the consequences. A real case study of utilizing decision tree will be discussed as follows:

In this work, we apply the decision tree analysis on real case study of an international company which makes gas compression plants all over the world and guarantees its plants availability. Therefore, it is important to make risk-informed decisions on the maintenance strategies that minimize the unavailability and downtime costs.

The case study is a site composed of two plants which is scratched in the following scheme at Fig. 1.

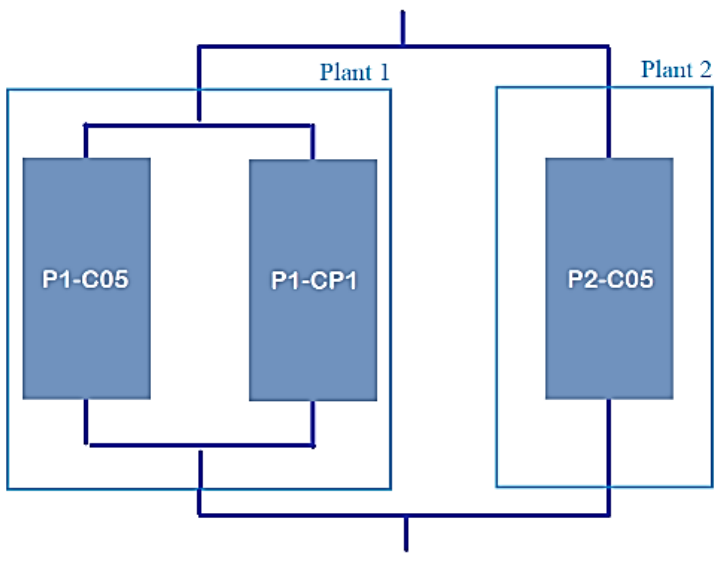

Fig.1. Scheme of the two plants, P1 and P2. P1 has two compressors $\mathrm{C} 05$ and $\mathrm{CP} 1$ in parallel, while $\mathrm{P} 2$ has one compressor C05. P2C05 can work instead of P1C05.

There are two plants Plant 1 and Plant 2 (called P1 and $\mathrm{P} 2$ respectively). $\mathrm{P} 1$ is composed of two different compressors $\mathrm{C} 05$ and $\mathrm{CP} 1$ in parallel compressing two different products. $\mathrm{P} 2$ is composed of just one compressor, the same as $\mathrm{P} 1$, the $\mathrm{C} 05$ compressor. In case of any accident and unavailability, $\mathrm{P} 2-\mathrm{C} 05$ can produce instead of P1-C05. Unavailability of P1 costs $10 \mathrm{~K} € /$ day while unavailability of $\mathrm{P} 2$ costs $6 \mathrm{~K} € /$ day. Every compressor consists of several subcomponents all working in series. In detail, C05 and CP1 have 27 subcomponents. We assume the subcomponents to be different in the case of the two compressors, due to different type of product.

The details of the probability of failure, unavailability, repair time, and the cost of the spares for each subcomponent are provided at Tables 1 to 3 . There are four qualitative levels defined for the failure probability of each subcomponent (Not Significant (NS), Low, Medium, High). The time of unavailability of the plant in case of failure of any subcomponent is related to the type of the subcomponent failed and the availability of the spares. For example, if breaker of the C05 compressor fails, the unavailability of plant $\mathrm{P} 1$ is 45 days in case there is no spares available. However, if the spares are already available, the unavailability of the plant 1 is just 8 hours that we call this unavailability of the repair time. If any other components of P1 fails, the unavailability of P1 is different from that of breaker and is specific for that failed subcomponent where their specific details can be read from Tables 1 to 3 .

Table 1. Subcomponents of plant P1-C05 (detailed properties)

\begin{tabular}{|c|c|c|c|c|c|}
\hline $\begin{array}{c}\text { Main } \\
\text { Equipment }\end{array}$ & Subcomponent & $\begin{array}{c}\text { Probability } \\
\text { of Failure }\end{array}$ & Unavailability & $\begin{array}{c}\text { Spare } \\
\text { price } \\
(\mathbf{k} €)\end{array}$ & $\begin{array}{c}\text { Repair } \\
\text { Time }\end{array}$ \\
\hline \multirow{2}{*}{ Breaker } & Breaker & Low & 1,5 months & 21 & 8 hours \\
\cline { 2 - 6 } & Protection relay & Low & 1 month & 6 & 8 hours \\
\hline \multirow{2}{*}{$\begin{array}{c}\text { Motor } \\
10 \mathrm{MW}\end{array}$} & Stator & Low & 2 months & 360 & 1 week \\
\cline { 2 - 6 } & Rotor & Low & 2 months & 240 & 1 week \\
\hline
\end{tabular}




\begin{tabular}{|c|c|c|c|c|c|}
\hline $\begin{array}{c}\text { Main } \\
\text { Equipment }\end{array}$ & Subcomponent & $\begin{array}{c}\text { Probability } \\
\text { of Failure }\end{array}$ & Unavailability & $\begin{array}{c}\text { Spare } \\
\text { price } \\
(\mathrm{k} €) \\
\end{array}$ & $\begin{array}{c}\text { Repair } \\
\text { Time }\end{array}$ \\
\hline & Bearing & Medium & 3 weeks & 24 & 1 week \\
\hline & Exchanger & NS & 2 weeks & 78 & 2 days \\
\hline Coupling & - & Low & 20 months & 48 & 1 week \\
\hline \multirow{2}{*}{ Gearbox } & Bull Gear & Low & 20 months & 210 & 2 weeks \\
\hline & Pignon-Shaft & Low & 20 months & 108 & 2 weeks \\
\hline \multirow{3}{*}{ Rotor } & Impeller & Low & 20 months & 42 & 2 weeks \\
\hline & Bearing & Low & 2 months & 24 & 5 days \\
\hline & $\begin{array}{l}\text { Labyrinths', } \\
\text { Seals }\end{array}$ & Low & 2 weeks & 15 & 2 weeks \\
\hline \multirow{2}{*}{ Exchanger } & Tube bundle & High & 2 weeks & 36 & 3 days \\
\hline & Shell & Low & NS & 48 & 3 days \\
\hline \multirow{2}{*}{ IGV } & Servomotor & Medium & 1 week & 9 & 4 hours \\
\hline & Nozzle & NS & 3 weeks & 18 & 2 days \\
\hline $\begin{array}{l}\text { Check- } \\
\text { Valve }\end{array}$ & - & Medium & 1 week & 3 & 1 days \\
\hline \multirow{4}{*}{ Anti-surge } & Valve & NS & 3 weeks & 6 & 6 hours \\
\hline & Servomotor & Medium & 1 week & 6 & 6 hours \\
\hline & Solenoid & Low & $8 \mathrm{~h}$ & 0.6 & 3 hours \\
\hline & Transmitter & Low & $8 \mathrm{~h}$ & 0.6 & 3 hours \\
\hline Vent Valve & - & Medium & 2 weeks & 7.2 & 6 hours \\
\hline PLC & - & Low & 2 weeks & 9 & 6 hours \\
\hline \multirow{4}{*}{ Oil circuit } & $\begin{array}{c}\text { Oil pump } \\
\text { trained }\end{array}$ & Medium & 1 month & 6 & 6 hours \\
\hline & $\begin{array}{l}\text { Oil pump } \\
\text { auxiliary }\end{array}$ & NS & 1 week & 9 & 6 hours \\
\hline & Exchanger & NS & 1 weeks & 9 & 8 hours \\
\hline & Oil filter & Low & NS & 2.4 & 2 hours \\
\hline
\end{tabular}

\begin{tabular}{|c|c|c|c|c|c|}
\hline $\begin{array}{c}\text { Main } \\
\text { Equipment }\end{array}$ & Subcomponent & $\begin{array}{c}\text { Probability } \\
\text { of Failure }\end{array}$ & Unavailability & $\begin{array}{c}\text { Spare } \\
\text { price } \\
\text { (k€) }\end{array}$ & $\begin{array}{c}\text { Repair } \\
\text { Time }\end{array}$ \\
\hline \multirow{4}{*}{ Anti-surge } & Valve & NS & 3 weeks & 15 & 6 hours \\
\cline { 2 - 7 } & Servomotor & Medium & 1 week & 15 & 6 hours \\
\cline { 2 - 7 } & Solenoid & Low & $8 \mathrm{~h}$ & 1.5 & 3 hours \\
\cline { 2 - 7 } & Transmitter & Low & $8 \mathrm{~h}$ & 1.5 & 3 hours \\
\hline Vent Valve & - & Medium & 2 weeks & 18 & 6 hours \\
\hline \multirow{2}{*}{ PLC } & - & Low & 2 weeks & 22.5 & 6 hours \\
\hline \multirow{3}{*}{ Oil circuit } & $\begin{array}{c}\text { Oil pump } \\
\text { trained }\end{array}$ & Medium & 1 month & 15 & 6 hours \\
\cline { 2 - 7 } & Oil pump & NS & 1 week & 22.5 & 6 hours \\
\cline { 2 - 7 } & Exchanger & NS & 1 weeks & 22.5 & 8 hours \\
\cline { 2 - 7 } & Oil filter & Low & NS & 6 & 2 hours \\
\hline
\end{tabular}

Table 3. Subcomponents of plant P2-C05 (detailed properties)

Table 2. Subcomponents of plant P1-CP1 (detailed properties)

\begin{tabular}{|c|c|c|c|c|c|}
\hline $\begin{array}{c}\text { Main } \\
\text { Equipment }\end{array}$ & Subcomponent & $\begin{array}{c}\text { Probability } \\
\text { of Failure }\end{array}$ & Unavailability & $\begin{array}{c}\text { Spare } \\
\text { price } \\
(\mathbf{k} \boldsymbol{\epsilon})\end{array}$ & $\begin{array}{c}\text { Repair } \\
\text { Time }\end{array}$ \\
\hline \multirow{3}{*}{ Breaker } & Breaker & Low & 1,5 months & 52.5 & 8 hours \\
\cline { 2 - 6 } & Protection relay & Low & 1 month & 15 & 8 hours \\
\hline \multirow{4}{*}{$\begin{array}{c}\text { Motor } \\
\text { 20MW }\end{array}$} & Stator & Low & 2 months & 900 & 1 week \\
\cline { 2 - 6 } & Rotor & Low & 2 months & 600 & 1 week \\
\cline { 2 - 6 } & Bearing & Medium & 3 weeks & 60 & 1 week \\
\cline { 2 - 6 } & Exchanger & NS & 2 weeks & 195 & 2 days \\
\hline Coupling & - & Low & 20 months & 120 & 1 week \\
\hline \multirow{3}{*}{ Gearbox } & Bull Gear & Low & 20 months & 525 & $\begin{array}{c}2 \\
\text { weeks }\end{array}$ \\
\cline { 2 - 6 } & Pignon-Shaft & Low & 20 months & 270 & $\begin{array}{c}2 \\
\text { weeks }\end{array}$ \\
\hline \multirow{4}{*}{ Rotor } & Impeller & Low & 20 months & 105 & $\begin{array}{c}2 \\
\text { weeks }\end{array}$ \\
\cline { 2 - 6 } & Bearing & Low & 2 months & 60 & 5 days \\
\cline { 2 - 6 } & labyrinths', & Low & 2 weeks & 37.5 & $\begin{array}{c}2 \\
\text { weeks }\end{array}$ \\
\hline \multirow{2}{*}{ Exchanger } & Tube bundle & High & 2 weeks & 90 & 3 days \\
\cline { 2 - 6 } & Shell & Low & NS & 120 & 3 days \\
\hline \multirow{2}{*}{ IGV } & Servomotor & Medium & 1 week & 22.5 & 4 hours \\
\cline { 2 - 6 } & Nozzle & NS & 3 weeks & 45 & 2 days \\
\hline $\begin{array}{c}\text { Check- } \\
\text { Valve }\end{array}$ & - & Medium & 1 week & 7.5 & 1 days \\
\hline \multirow{2}{*}{} & & & & \\
\hline
\end{tabular}

\begin{tabular}{|c|c|c|c|c|c|}
\hline $\begin{array}{c}\text { Main } \\
\text { Equipment }\end{array}$ & Subcomponent & $\begin{array}{c}\text { Probability } \\
\text { of Failure }\end{array}$ & Unavailability & $\begin{array}{c}\text { Spare } \\
\text { price } \\
(\mathbf{k} \epsilon)\end{array}$ & $\begin{array}{c}\text { Repair } \\
\text { Time }\end{array}$ \\
\hline \multirow{2}{*}{ Breaker } & Breaker & Low & 1,5 months & 35 & 8 hours \\
\hline & Protection relay & Low & 1 month & 10 & 8 hours \\
\hline \multirow{4}{*}{$\begin{array}{l}\text { Motor } \\
20 \mathrm{MW}\end{array}$} & Stator & Low & 2 months & 600 & 1 week \\
\hline & Rotor & Low & 2 months & 400 & 1 week \\
\hline & Bearing & Medium & 3 weeks & 40 & 1 week \\
\hline & Exchanger & NS & 2 weeks & 130 & 2 days \\
\hline Coupling & - & Low & 20 months & 80 & 1 week \\
\hline \multirow{2}{*}{ Gearbox } & Bull Gear & Low & 20 months & 350 & $\begin{array}{c}2 \\
\text { weeks } \\
\end{array}$ \\
\hline & Pignon-Shaft & Low & 20 months & 180 & $\begin{array}{c}2 \\
\text { weeks }\end{array}$ \\
\hline \multirow{3}{*}{ Rotor } & Impeller & Low & 20 months & 70 & $\begin{array}{c}2 \\
\text { weeks }\end{array}$ \\
\hline & Bearing & Low & 2 months & 40 & 5 days \\
\hline & $\begin{array}{c}\text { labyrinths', } \\
\text { Seals }\end{array}$ & Low & 2 weeks & 25 & $\begin{array}{c}2 \\
\text { weeks }\end{array}$ \\
\hline \multirow{2}{*}{ Exchanger } & Tube bundle & High & 2 weeks & 60 & 3 days \\
\hline & Shell & Low & NS & 80 & 3 days \\
\hline \multirow{2}{*}{ IGV } & Servomotor & Medium & 1 week & 15 & 4 hours \\
\hline & Nozzle & NS & 3 weeks & 30 & 2 days \\
\hline $\begin{array}{c}\text { Check- } \\
\text { Valve }\end{array}$ & - & Medium & 1 week & 5 & 1 days \\
\hline \multirow{4}{*}{ Anti-surge } & Valve & NS & 3 weeks & 10 & 6 hours \\
\hline & Servomotor & Medium & 1 week & 10 & 6 hours \\
\hline & Solenoid & Low & $8 \mathrm{~h}$ & 1 & 3 hours \\
\hline & Transmitter & Low & $8 \mathrm{~h}$ & 1 & 3 hours \\
\hline Vent Valve & - & Medium & 2 weeks & 12 & 6 hours \\
\hline PLC & - & Low & 2 weeks & 15 & 6 hours \\
\hline \multirow{4}{*}{ Oil circuit } & $\begin{array}{c}\text { Oil pump } \\
\text { trained }\end{array}$ & Medium & 1 month & 10 & 6 hours \\
\hline & $\begin{array}{l}\text { Oil pump } \\
\text { auxiliary }\end{array}$ & NS & 1 week & 15 & 6 hours \\
\hline & Exchanger & NS & 1 weeks & 15 & 8 hours \\
\hline & Oil filter & Low & NS & 4 & 2 hours \\
\hline
\end{tabular}

There is the above-mentioned information available in the uncertain environment. The company wants to get risk-informed recommendations to decide 
to buy which spare parts to reduce the unavailability of the plant. The decision tree provides a useful tool for the company to decide in a rational way being informed of the risks and benefits of each action. Therefore, to give reliable information to the decision maker to buy or not to buy each specific subcomponent, a decision tree will be drawn for each of $3 * 27$ subcomponents integrating the unavailability and repair cost with its associated failure probability to give him/her an expected value of taking each action in monetary terms. This utility value will give a comparison tool for the decision maker to choose the most profitable action.

The decision analysis is performed based on two different approaches. In the first approach that is called one site approach, a decision tree is drawn for each subcomponent failure and the unavailability of the plant imposed by failure is compared to the no failure event in two different strategies of buying and not buying the spare parts. The expected values derived from the decision tree will help the decision maker to find the convenient strategy for that subcomponent. The second approach is called the multi-site approach in which the unavailability of P1-C05 compressor can be replaced by a fraction with the P2-C05 compressor. These approaches are described in the following sections.

\section{One Site Approach}

The failure probability of each subcomponent is defined qualitatively. First, a quantitative value is dedicated to each of them. The quantitative value of the probability of failure is derived from the frequentist definition of probability [25] where the probability of failure of a component in its lifetime comes from the number of failures experienced from past operational experience. As an example, assuming there is enough data of the past operational experience for a specific component, when in a sample of 10000 components working at the same operational stress, 1 failure has occurred during that components standard lifetime, the probability of failure is resulted to be equal to 0.0001 . This quantity is assumed as the value of the probability of failure for NS qualitative category. Similarly, we assume that the Low probability of a component corresponds to a probability of failure equal to 0.01 , Medium probability to 0.03 , and finally High probability to 0.1 . In addition, we know the compressor $\mathrm{P} 1-\mathrm{C} 05$ is producing the $60 \%$ of the capacity, so we assume that its cost of unavailability is equal to $0.6 * 10 \mathrm{~K} € /$ day, while the cost of unavailability of P1-CP1 is $0.4 * 10 \mathrm{~K} € /$ day.

The example decision tree of Fig. 2 is for the breaker subcomponent of the breaker equipment of plant P1-C05 with the properties indicated at the second row of Table 1. There are two actions available for the company. First, is to buy the spare and second is to do nothing. Each action has its own consequences in terms of cost (namely the cost of the spare part or the cost unavailability if a failure occurs). There are also two probable outcomes after the action is decided which is the failure or nofailure of the component. For example, for breaker subcomponent of P1-C05, the probability of failure $\left(\mathrm{P}_{\mathrm{f}}\right)$ equals to $3 \%$. The spare cost is $21 \mathrm{~K} €$. The unavailability of the plant in case of no spare is 45 days with the cost equal to $45^{*} 6 \mathrm{~K} €$, and the repair time is 8 hours with the cost equal to $8 / 24 * 6 \mathrm{~K} €$. There are 4 possible scenarios:

1. The action is to do nothing, and the subcomponent fails. This means that to take the plant back to operation, the total cost is to pay for the unavailability of the plant in 45 days $\left(45^{*} 6 \mathrm{~K} €\right)$ plus the spare part.

2. The action is to do nothing, and the subcomponent does not fail. This is the ideal scenario in which the cost is zero.

3. The action is to buy the spare, and the subcomponent fails. This means that to take the plant back to operation, the total cost is to pay for the unavailability of the plant (i.e. the repair time) in 8 hours $(8 / 24 * 6 \mathrm{~K} €)$ plus the spare part (spare part cost was paid before the failure occurred).

4. The action is to buy the spare, and the subcomponent does not fail. In this case, only the spare part cost is paid.

These four scenarios are schemed at the decision tree of Fig. 2, where the values beside each scenario's end state (i.e. the red triangle) is its consequent cost and scenario's number is drawn inside the triangle.

First, to find the expected loss of each scenario, the cost of scenario is multiplied by its associated probability. Therefore, the expected loss of each scenario is calculated as follows:

1. $\mathrm{S}_{1}=$ expected loss for scenario $1=291 \times 0.03$

2. $\mathrm{S}_{2}=$ expected loss for scenario $2=0$

3. $\mathrm{S}_{3}=$ expected loss for scenario $3=23 \times 0.03$

4. $\mathrm{S}_{4}=$ expected loss for scenario $4=21 \times 0.97$

Then the expected loss of each action is calculated as the summation of its relevant scenarios. If the action is to do nothing, there are two probable scenarios of failure and no failure (scenarios 1 and 2). Therefore, the expected loss is equal to summation of expected loss for failure $\left(S_{1}\right)$ plus the expected loss for no failure $\left(S_{2}\right)$, making the expected loss of doing nothing $\left(\mathrm{A}_{1}\right)$ equal to:

- $\mathrm{A}_{1}=\mathrm{S}_{1}+\mathrm{S}_{2}=8.73$

Similarly, the expected loss of the action "buy spares" $\left(\mathrm{A}_{2}\right)$ equals to the summation of expected losses of scenarios 3 and 4

- $\mathrm{A}_{2}=\mathrm{S}_{3}+\mathrm{S}_{4}=21.06$

Comparing these expected losses associated with each action $\left(A_{1}\right.$ and $\left.A_{2}\right)$, it is concluded that the action of do nothing $\left(\mathrm{A}_{1}\right)$ has lower expected loss than buying spare $\left(A_{2}\right)$. Therefore, the rational decision here is to do nothing 
for the breaker since it minimizes the loss and in other word maximizes the benefit.

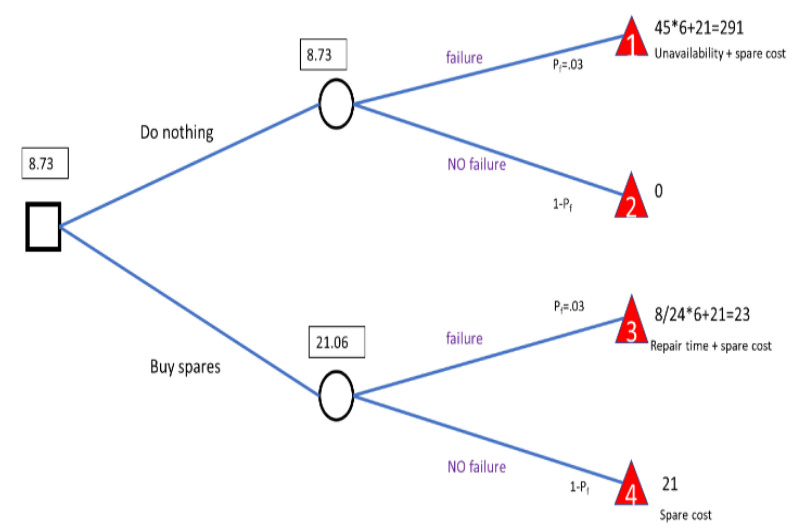

Fig.2. Decision tree of each subcomponent of each compressor. The compressors are considered as independent. The negative sign for costs is omitted.

Similarly, the decision tree of Fig. 2. were drawn for every subcomponent of each compressor to decide whether it is profitable to buy the spare parts or not. Thanks to the $27 \times 3$ decision trees drawn for all the subcomponents shown in Tables 1 to 3 , the expected losses of doing nothing and buying the spares are calculated for all of them. The rational decisions are deducted. Those subcomponents that need the spares to be bought in advance, based on the decision tree analyses, are listed in Table 4.

Table 4. Sub-Components that need buying spares

\begin{tabular}{|c|c|c|c|}
\hline Subcomponent & $\begin{array}{c}\text { Expected loss } \\
\text { for doing } \\
\text { nothing }\end{array}$ & $\begin{array}{c}\text { Expected loss } \\
\text { for Buying } \\
\text { spares }\end{array}$ & Delta \\
\hline ImpellerP1C05 & 109.26 & 44.52 & 64.74 \\
\hline CouplingP1C05 & 109.44 & 49.26 & 60.18 \\
\hline ImpellerP2C05 & 110.1 & 72.52 & 37.58 \\
\hline CouplingP2C05 & 110.4 & 81.26 & 29.14 \\
\hline $\begin{array}{c}\text { Oil pump } \\
\text { trainedP1C05 }\end{array}$ & 9.3 & 6.075 & 3.225 \\
\hline Pignon-ShaftP1C05 & 111.24 & 110.52 & 0.72 \\
\hline
\end{tabular}

The results of Table 4 shows that out of 81 subcomponents of 3 plants, it is rational to buy the spares for the 6 listed subcomponents. The Ranking of the difference among the expected losses of subcomponents are also provided with the Delta value, the greater delta is the greater value of buying spare. Based on this ranking and in case of limited budget, the company can choose which subcomponents to buy first with the greater delta value.

\section{Multi-site Approach}

Since plant 1 is composed of two compressors, its availability is more important than plant 2 in terms of the product value. Moreover, plant 1 and 2 share the same compressors (C05) in two different sites. Same subcomponents are also shared by both P1C05 and P2C05 and the unavailability cost of $\mathrm{P} 1 \mathrm{C} 05$ and $\mathrm{P} 2 \mathrm{C} 05$ is the same. Therefore, we consider the case where there is the possibility to make P2-C05 work instead of P1-C05 in case of P1-C05 unavailability.

The idea is to suppose that a percentage $t$ of the production of $\mathrm{P} 1-\mathrm{C} 05$ can be assigned to $\mathrm{P} 2-\mathrm{C} 05$ in case of emergency. This enables the plant 1 to maintain production and cause a decrease of the cost of unavailability of $\mathrm{P} 1$ to $(1-\mathrm{t}) * 6 \mathrm{~K} € /$ day, assuming that $\mathrm{P} 2$ is not full capacity.

The decision tree of Fig. 3 describes the process, considering both $\mathrm{P} 1-\mathrm{C} 05$ and $\mathrm{P} 2-\mathrm{C} 05$. In total 27 decision trees will be drawn for all subcomponents of P1-C05.It worth mentioning that this approach is focused on keeping plant 1 operational and the operation of plant 2 will be sacrificed for plant 1 . Two forms of probability of failure is used in this approach: First, the probability of the failure of the subcomponents of P1-C05 which is similar to the on-site approach and is derived from Table 1 (denoted as failure or its complements NO failure in Fig. 3). Second, the probability of the operation (reliability) of plant 2 (denoted as P2 works in Fig. 3) depends on the operation of all its subcomponents and is derived from Equation 1:

$$
R_{2}=\prod_{i=1}^{27}\left(1-p_{i}\right)
$$

Where $p_{i}$ is the probability of failure of each subcomponent of plant 2 derived from Table 3 . This means that for plant 2 to be operational, all its 27 subcomponents should be operational.

At the other hand, the failure probability of $\mathrm{P} 2-\mathrm{C} 05$ is simply the complement of $\mathrm{R}_{2}$ (denoted as P2 does not work in Fig. 3) which is equal to the equation:

$P_{f 2}=1-R_{2}$

We assume the probability of simultaneous failure for same subcomponents of P2-C05 and P1-C05 is zero. The costs are only computed in terms of $\mathrm{P} 1$; thus, we are not computing the cost of repairing $\mathrm{P} 2$.

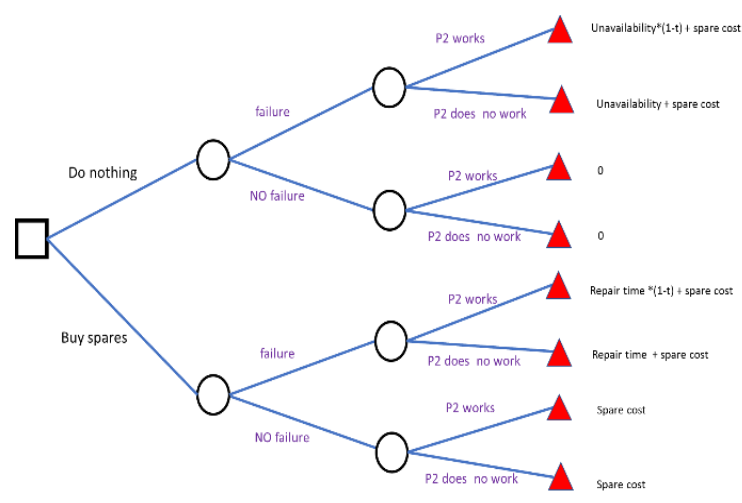

Fig. 3. Multiple Plants decision tree for P1-C05. Computing the cost of P1 since P2-C05 can partially work instead of P1C05.

For each subcomponent of P1-C05 the decision tree can be drawn with this logic that if the subcomponent fails, the unavailability of P1-C05 is not $100 \%$ and a 
fraction $\mathrm{t}$ of its unavailability can be compensated by using the products of plant 2 as shown in Fig. 3.

For every subcomponent, we can plot the estimated expected loss of the action "do nothing" in terms of percentage of production added to $\mathrm{P} 2-\mathrm{C} 05$ in case of P1C05 failure. Fig. 4 depicts this for the "Coupling" sub equipment of $\mathrm{P} 1-\mathrm{C} 05$. When $\mathrm{t}=0$, the expected loss is equal to the one site approach where its values can be referred from Table 4. We can see in this figure that when $t$ increases the expected loss of do nothing" decreases, and therefore, there is no need to buy the spares.

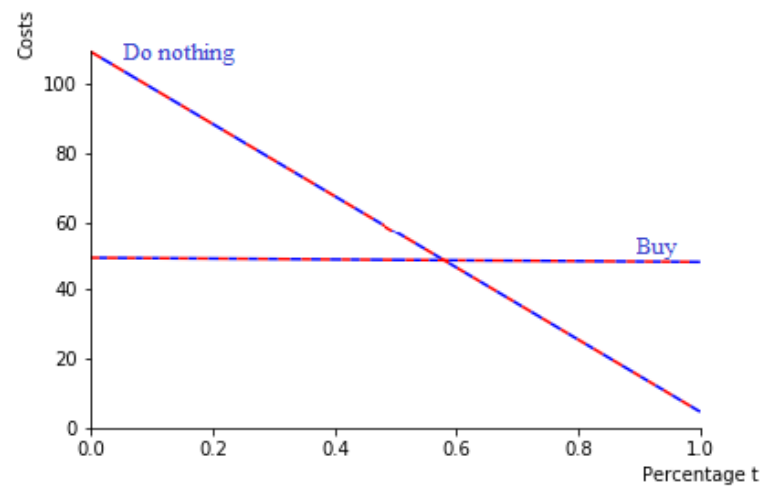

Fig. 4. Subcomponent Coupling of P1-C05 Compressor. Expected value if spare is bought in advance or not.

For three levels of $\mathrm{t}$, namely $0.2,0.5$, and 0.8 , The decision trees are computed for each subcomponent of P1$\mathrm{C} 05$, and the expected losses of two strategies of buying spares and doing nothing are calculated for the three mentioned $\mathrm{t}$ values. Under these assumptions, it always better not to buy subcomponents when $\mathrm{t}=0.8$, buy two subcomponents when $\mathrm{t}=0.5$, and buy three subcomponents when $\mathrm{t}=0.2$. Table 5 summarizes the results.

Table 5. Subcomponents that need buying spares

\begin{tabular}{|c|c|c|}
\hline $\mathbf{t}=\mathbf{0 . 2}$ & $\mathbf{t}=\mathbf{0 . 5}$ & $\mathbf{t = 0 . 8}$ \\
\hline ImpellerP1C05 & ImpellerP1C05 & - \\
\hline CouplingP1C05 & CouplingP1C05 & - \\
\hline $\begin{array}{c}\text { Oil pump } \\
\text { trainedP1C05 }\end{array}$ & - & - \\
\hline
\end{tabular}

Results show that when the t percentage increases, there is no need to buy the spares since the unavailability is better compensated by the plant 2 operation. However, when $\mathrm{t}$ is lower, there are more subcomponents needed to be bought.

\section{Conclusion}

In this research, using the decision analysis, a simple vulnerability analysis of the gas production site is conducted. The decision trees are computed for two approaches of one site and multi-site. Recommendation lists for the subcomponents that their spares should be bought are provided both for the one site and multi-site approach. It is concluded that the subcomponent Impeller and Coupling are the most vulnerable subcomponents and their failure impose high risk and cost to the system. In the multi-site approach, it is concluded that when the fraction of production replacement by the other plant increase, the need for buying spare reduces. Utilizing a fraction of $\mathrm{P} 2$ compressor's production when $\mathrm{P} 1$ fails can help saving money in terms of unavailability of P1C05.The framework proposed in this work is a sophisticated approach while it is feasibly applicable to the industry and can be applied on real case studies of industrial systems to give them risk informed decisions on maintenance strategy while reliably capturing a good prediction on their systems availability.

\section{References}

[1] M.H. Faber, Statistics and probability theory: in pursuit of engineering decision support, Vol. 18., London: Springer Science \& Business Media, 2012.

[2] J.-T. Horn and B. J. Leira., "Fatigue reliability assessment of offshore wind turbines with stochastic availability," Reliability Engineering \& System Safety, vol. 191, pp. 17, 2019.

[3] H.L.J. Rivera-Gómez, O. Montaño-Arango, E. HernándezGress, J. Corona-Armenta and F. Santana-Robles, "Joint production and repair efficiency planning of a multiple deteriorating system," Flexible Services and Manufacturing Journal, vol. 31, no. 2, p. 446-471, 2018.

[4] P. Pagani, G. Fischer, I. Farquhar, R. Skilton and M. Mittwollen, "A logistical simulation tool to quantitatively evaluate the effect of different maintenance solutions on the total maintenance downtime for fusion reactors," Fusion Engineering and Design, vol. 141, pp. 121-124, 2019.

[5] R. Manzini, A. Regattieri, H. Pham and E. Ferrari, Maintenance for industrial systems, London: Springer Science \& Business Media, 2009.

[6] J. Moubray, Reliability-centered maintenance, Amsterdam: Industrial Press Inc., 2001.

[7] R.A. Howard, "Information value theory," IEEE Transactions on systems science and cybernetics, vol. 2, no. 1, pp. 22-26, 1966.

[8] D. Straub, "Value of information analysis with structural reliability methods," Structural Safety, vol. 49 , pp. 75-85, 2014.

[9] H. Raiffa and R. Schlaifer, Applied Statistical Decision Theory, Harvard University, 1961.

[10] M. H. DeGroot, Optimal statistical decisions, Vol. 82: ohn Wiley \& Sons, 2005.

[11] M. H. Faber and R. Rackwitz, "Sustainable decision making in civil engineering," Structural Engineering International, vol. 14, no. 3, pp. 237-242, 2004. 
[12] E. Borgonovo, V. Cappelli, F. Maccheroni and M. Marinacci, "Risk analysis and decision theory: A bridge," European Journal of Operational Research, vol. 264, no. 1, pp. 280-293, 2018.

[13] B. Adem Esmail and D. Geneletti, "Multi-criteria decision analysis for nature conservation: A review of 20 years of applications," Methods in Ecology and Evolution, vol. 9, no. 1, pp. 42-53, 2018.

[14] J. G. Thornton, R. Lilford and N. Johnson, "Decision analysis in medicine," BMJ: British Medical Journal, vol. 304, no. 6834, p. 1099, 1992.

[15] D. M. Logan, " Decision analysis in engineering-economic modeling," Energy, vol. 15, no. 7, pp. 677-696, 1990.

[16] S. Thöns and M. H. Faber, "Assessing the value of structural health monitoring ().," ICOSSAR'13 - 11th International conference on structural safety and reliability - Safety, reliability, risk and life-cycle performance of structures and infrastructures (Proceedings), 2013.

[17] D. Zonta, B. Glisic and S. Adriaenssens, "Value of information: impact of monitoring on decision-making," Structural Control and Health Monitoring, vol. 21, no. 7, pp. 1043-1056, 2014.

[18] C. Malings and M. Pozzi, "Value of Information for Spatially Distributed Systems: application to sensor placement," Reliability Engineering \& System Safety, vol. 154, pp. 219-233, 2016a.

[19] C. Malings and M. Pozzi, "Conditional entropy and value of information metrics for optimal sensing in infrastructure systems," Structural Safety, vol. 60, pp. 77-90, 2016 b.

[20] C. Malings and M. Pozzi, "Value-of-information in spatiotemporal systems: Sensor placement and scheduling," Reliability Engineering \& System Safety, vol. 172, pp. 4557, 2018

[21] S. R. Safavian and D. Landgrebe, "A survey of decision tree classifier methodology," IEEE transactions on systems, man, and cybernetics, vol. 21, no. 3, pp. 660-674, 1991.

[22] D. Straub and A. Der Kiureghian, "Bayesian network enhanced with structural reliability methods: Application," Journal of Engineering Mechanics, vol. 136, no. 10, pp. 1259-1270, 2010.

[23] J. R. Quinlan, "Induction of decision trees," Machine learning, vol. 1, no. 1, pp. 81-106, 1986.

[24] L. Rokach and O. Maimon, "Decision trees," in Data mining and knowledge discovery handbook, Boston, MA, Springer, 2005, pp. 165-192.

[25] E. Zio, An introduction to the basics of reliability and risk analysis. Vol. 13., London: World scientific, 2007. 